\title{
Sonder une collection
}

Tiziana N. Beltrame, Sophie Houdart, Christine Jungen et Frédéric Keck

\section{(2) OpenEdition \\ Journals}

Édition électronique

URL : https://journals.openedition.org/tc/8641

DOI : $10.4000 /$ tc. 8641

ISSN : 1952-420X

Éditeur

Éditions de l'EHESS

Édition imprimée

Date de publication : 18 décembre 2017

Pagination : 178-195

ISBN : 978-2-7132-2708-0

ISSN : 0248-6016

Référence électronique

Tiziana N. Beltrame, Sophie Houdart, Christine Jungen et Frédéric Keck, « Sonder une collection », Techniques \& Culture [En ligne], 68 | 2017, mis en ligne le 18 décembre 2019, consulté le 29 septembre 2022. URL : http://journals.openedition.org/tc/8641 ; DOI : https://doi.org/10.4000/tc.8641 


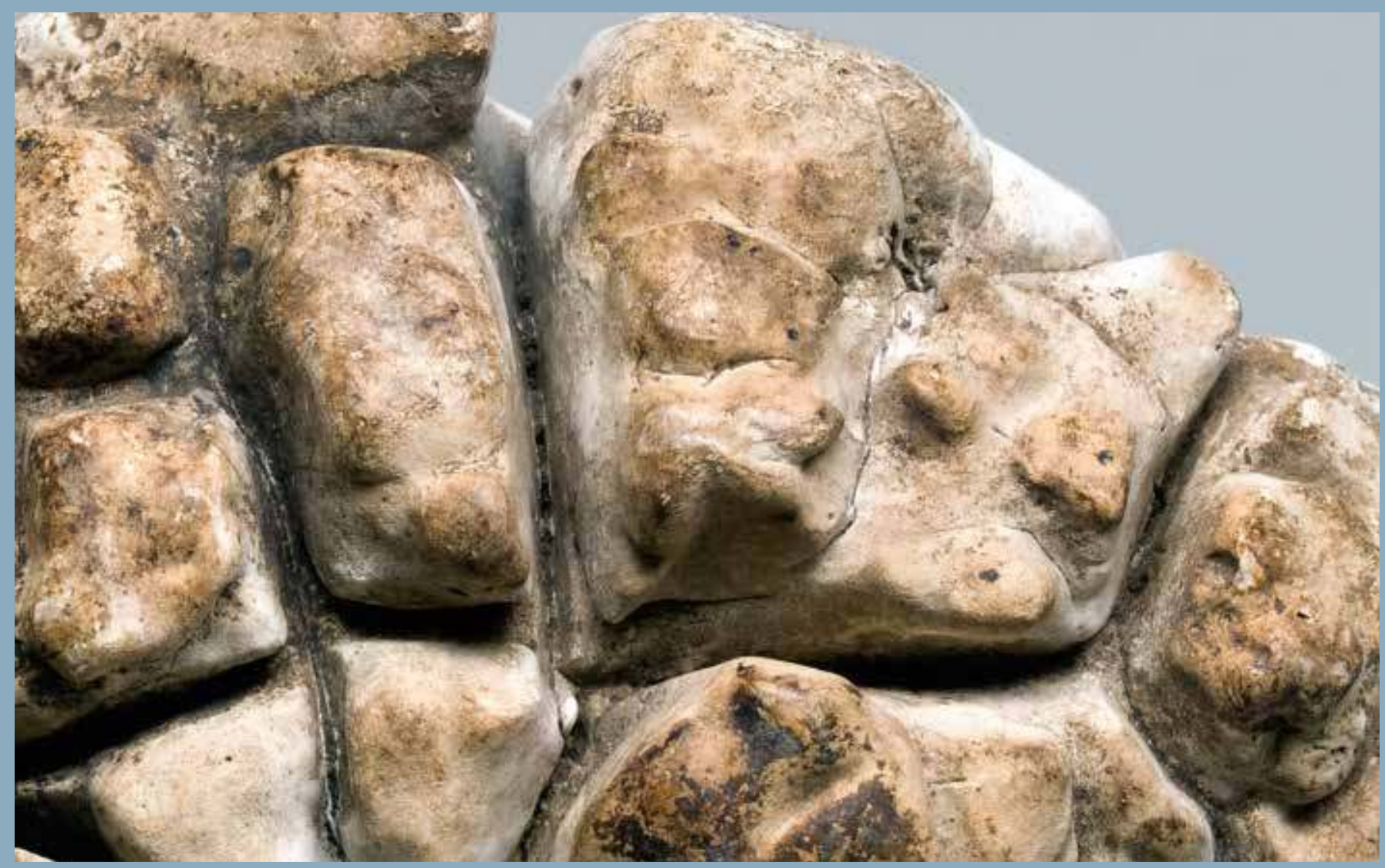




\section{Sonder une collection}

Soit un musée d'ethnographie. Ou plutôt, soit un musée des arts et civilisations qui a hérité de collections ethnographiques, rassemblées par des missionnaires, des voyageurs, des ethnographes. Ces collections sont le fruit d'une histoire particulière - celle qui a vu, dans le cadre de ce qui deviendra le musée de l'Homme, des missions scientifiques dépêchées dans le monde pour archiver l'homme dans l'ensemble de ses déclinaisons culturelles.

Soient ces collections d'objets ethnographiques. La partie visible, celle présentée dans les salles d'exposition, a été reformulée, dans le cadre du transfert du musée de l'Homme au musée du quai Branly, en collection d'objets d'art - arts premiers, arts des civilisations non européennes. Dans les réserves, ce qui reste: en sus des objets qui ont vocation à être exposés, des résidus, reliquats, débris des missions ethnographiques ou des collections personnelles assemblées par un voyageur curieux.

Soient quatre anthropologues, assis devant un terminal relié à la base de données interne du musée. Ils préparent une proposition d'exposition sur les «petits êtres » et les mondes que ceux-ci produisent, et explorent pour ce faire la base de données informatiques des collections TMS (The Museum System). Ils sondent, littéralement, le terrain, en lançant des mots-clés dans la base de données, essayant d'évaluer la morphologie des listes d'objets ainsi appariés qui émerge sous les coups de sonde.

Que voit-on quand on sonde la base de données par l'échelle du petit? Tout d'abord la série de traces, scrupuleusement conservées dans les réserves, de l'ambition initiale des ethnographes archiveurs: collecter, décrire, cataloguer, conserver jusqu'aux éléments les plus minimaux, les plus énigmatiques parfois. Aucun élément des collections du musée de l'Homme et du musée national des arts d'Afrique et d'Océanie n'a échappé au traitement du «chantier des collections» lors de leur transfert au musée du quai Branly. Ils ont été pour la plupart rattachés à un numéro d'inventaire, ainsi qu'à une fiche TMS, nettoyés, photographiés et conditionnés en vue de leur stockage dans les réserves. On trouve: des « débris» de cocons de vers à soie entassés dans une petite boîte ronde; de minuscules «éléments métalliques», conservés dans les sachets 
1. Échantillon de matière première, $\mathrm{n}^{\circ}$ inventaire 70.2004.3.81.

«Pâte brun foncé faite des graines de "chía" (plante) broyées.

A partir de cette pâte est probablement obtenue l'huile de Chía. Utilisé dans la fabrication des objets laqués » (Rubrique description et usage de la fiche $\mathrm{MH}$ et MQB). Claude et Guy Stresser-Péan. Collecte:

Arnaud Seydou. Provenance: Mexique.

2. Jeu de nouds de ficelle, $\mathrm{n}^{\circ}$ inventaire 71.1936 .48 .316 .

Objet du Brésil, mission Dina et Claude Lévi-Strauss, conservé au département Amérique du musée de l'Homme avant son transfert au musée du quai Branly.

Selon la rubrique «usage» de la fiche, ces fibres végétales constituent un «échantillonnage des différentes manières de nouer connues par les Boróro[a]. Exécutés par un homme». soigneusement numérotés; des fragments de terre cuite de taille similaire $-4 \times 3 \times 1 \mathrm{~mm}$ pour le plus grand, précise la notice - que la description ne sait nommer autrement que par «Divers». Des boîtes, des bouteilles, des sachets aussi, dans lesquels est contenu tout ce qui relève d'une morphologie ou d'une matérialité qui échappe à la main: magmas, poudres, perles, graines, cristaux.

L’entrée se fait par le fragmentaire, le granulaire, le fibreux, le pulvérisé: tessons, fragments, résidus, échantillons, poudres, poussières peuplent en masse la collection tout en gardant leur discrétion. Les objets s'animent lorsqu'on cherche par insectes, élytres, cochenilles. Des pots de fourmis et de collier d'élytres côtoient les traces d'autres petits êtres xylophages ou kératophages dont les larves laissent des trous à leur passage dans des sculptures en bois, des livres anciens, des figurines en biscuit. Oxydations, concrétions, fermentations, putréfactions, cristallisations, exsudations de quelques exemples d'objets, tel un exsudat de cochenille ou des patines calcaires d'une boule boleadora, montrent les processus à l'œuvre aux échelles microscopiques qui deviennent visibles à l'échelle humaine.

Les mots-clés soumis (grains - poussières - insectes - fibres - résidus - poudres - brins concrétions) font ainsi apparaître des séries hétéroclites, telle une chambre de merveilles qui constitue « une exploration jubilatoire de toutes les performances dont est capable une logique d'inventaire, une confrontation joueuse et désinvolte de tous les systèmes de classifications disponibles» (Falguières 2003: 42). Alors que la disposition et la dénomination de ces objets étaient issues du domaine des sciences naturelles qui reposaient sur des critères physiques observables, ces requêtes constituent aujourd'hui des entrées singulières et des récits disjoints, où le goût pour l'étrange et le merveilleux prédominent.

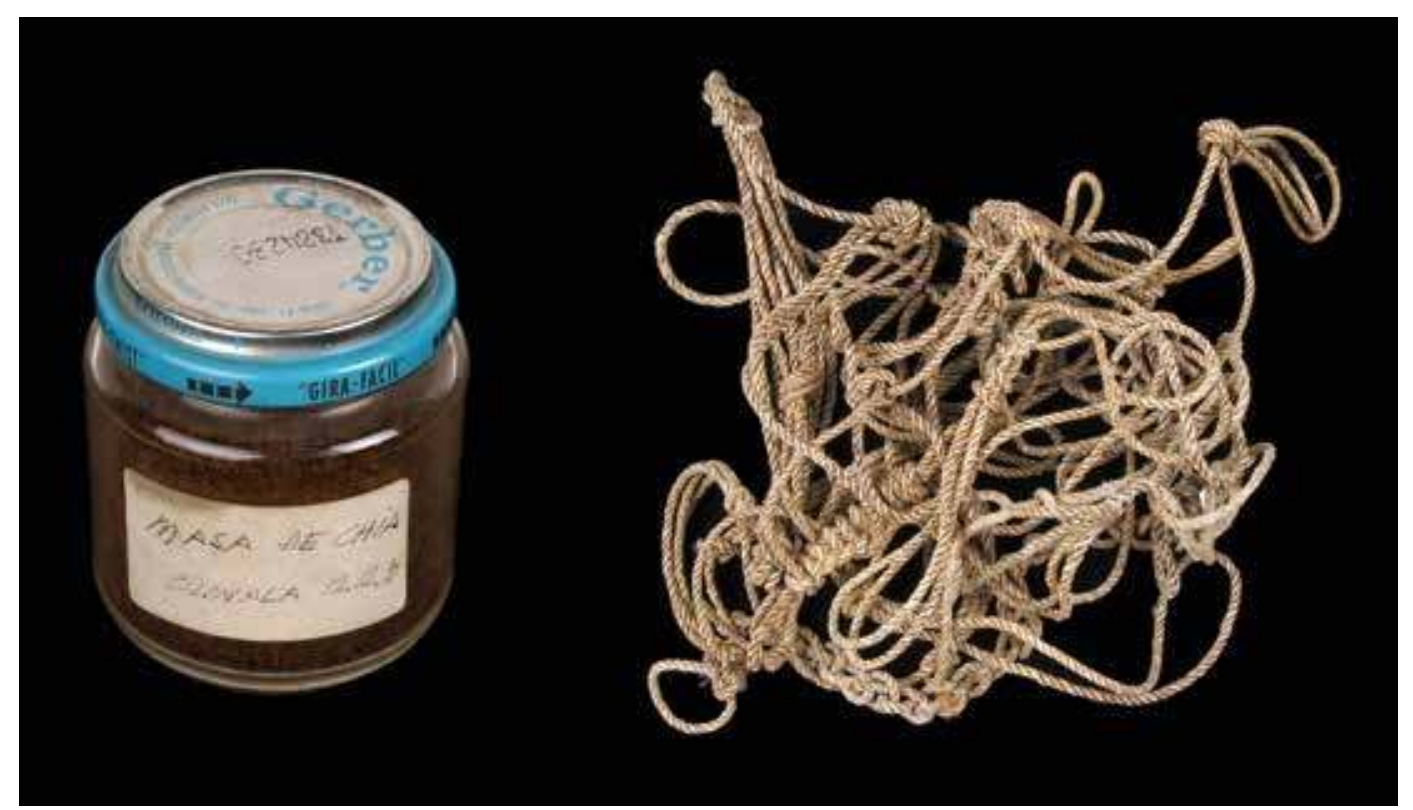


Par ces séries curieuses qu'il fait émerger, l'entrée par le petit constitue des «jeux de ficelle», pour emprunter à Donna Haraway (2016) : simultanément algorithmes, casse-tête, jeux de dextérité et diseurs d'histoires, les fils et leurs nœuds tissent et relient, combinent et connectent, racontent des manières inédites de «devenir avec » : soient ici, quatre entrées, quatre histoires, quatre récits à partir de l'infime dans les collections du musée du quai Branly.

\section{Insecte}

Face à l'ordinateur, j'entre dans la base de données du musée par le mot «insecte» et c'est un ensemble de pots et d'ornements composés de corpuscules ainsi que de dispositifs de captation de ces petits êtres qui apparaîtà l'écran: pièges à termites du Vietnam, cages à criquets de Bali, nattes à fourmis de Guyane. Les notices laissent deviner les usages de ces dispositifs de captation et imaginer le son des insectes dans leurs interactions avec les humains. Les éleveurs de criquets excitent les insectes au combat par une tige de graminée. La vannerie est enfoncée dans le sol pour que les termites s'y accumulent. La natte est posée sur la peau des initiés pour que les piqûres de fourmis les soumettent à une mise à l'épreuve de la douleur. Chacun de ces objets est un micromonde reliant des êtres à des échelles différentes; mais il faut pour le sentir dépasser le stade du visuel pour entrer dans une relation sonore et tactile avec ces petits êtres - peut-être même olfactive si l'on trouve des procédés de fermentation.

C'est alors tout le dispositif du musée qui apparaît lui-même comme un piège destiné à capter les présences des petits êtres: pièges aux phéromones sur le plateau des collections, système d'anoxie avant l'entrée dans les réserves... En collaboration avec la Cité de la Musique, le musée du quai Branly collabore au projet de recherche «Requiem pour Xylophages», qui permet l'application de procédés, notamment l'«ATAX» (Analyse des Traces Acoustiques de Xylophages), pour capter les craquements des fibres de bois produits par les insectes dans les objets. Parmi ceux-ci, les instruments de musique qui semblaient ne plus pouvoir produire de son du fait de leur muséification retrouvent ainsi une présence sonore du fait de leur environnement de conservation. Quand les insectes ne sont plus là, des trous attestent leur passage.

Une boîte de café ramenée par Marcel Griaule de la mission Dakar Djibouti, dont Julien Bondaz nous livre un beau récit d'« ethnographie parasitée» (2013), contient des fourmis sèches et produit donc un effet de gigogne ou de poupée russe: si le musée est lui-même une boîte contenant des insectes vivants et morts, la question est alors de savoir à quelle échelle se placer pour construire des interactions avec ces petits êtres. Matthieu Abonnenc conclut son film sur les traces de la mission Dakar Djibouti par les images de boîtes d'objets ouvertes dans le cadre du chantier de rénovation du musée de l'Homme et dont s'échappent des scarabées. L'héroïne du film, hantée par les enquêtes entomologiques de Griaule et Leiris, semble retrouver prise sur le réel en ouvrant ces boîtes dans un musée en rénovation. Chacun des objets du musée 


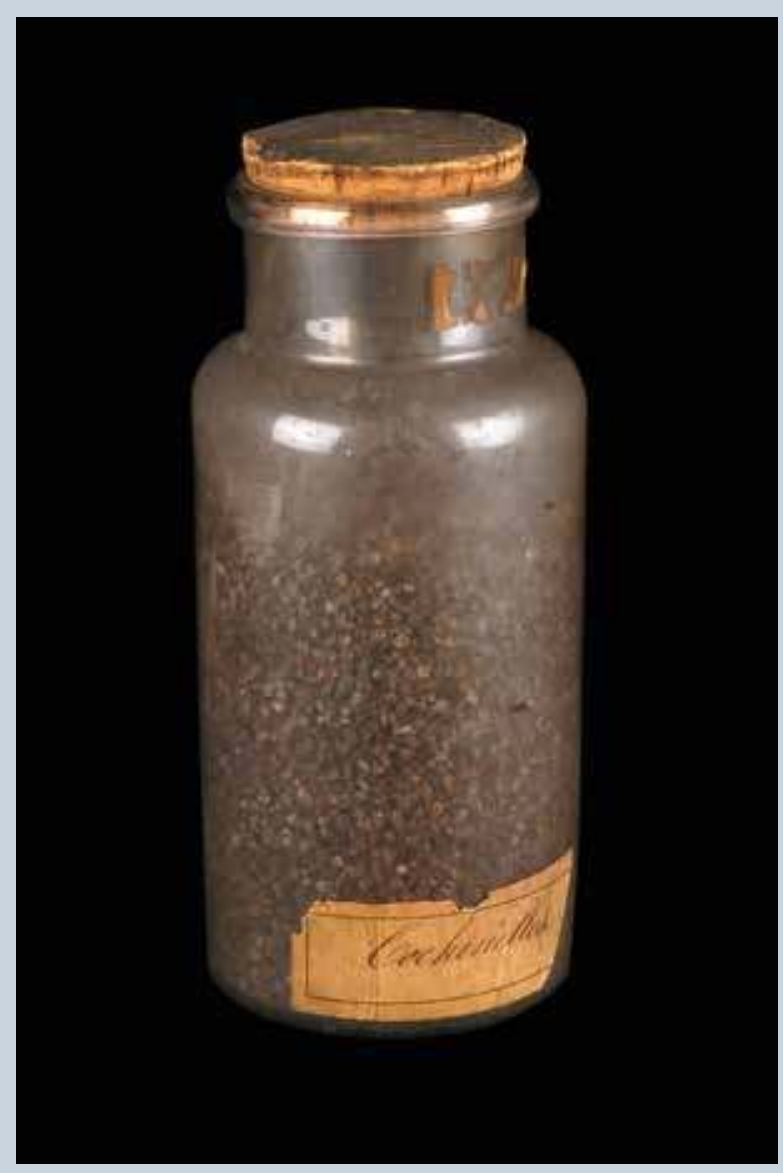

Pot de cochenille, $n^{\circ}$ inventaire 71.1950.81.6.3.

Cet objet a été donné par le Musée colonial de la ville de Lyon. Il a été inventorié au musée de l'Homme en 1950 et conservé au département de Technologie Comparée. La provenance des cochenilles n'étant pas identifiée, l'objet demeure hors Unité Patrimoniale au musée du quai Branly.

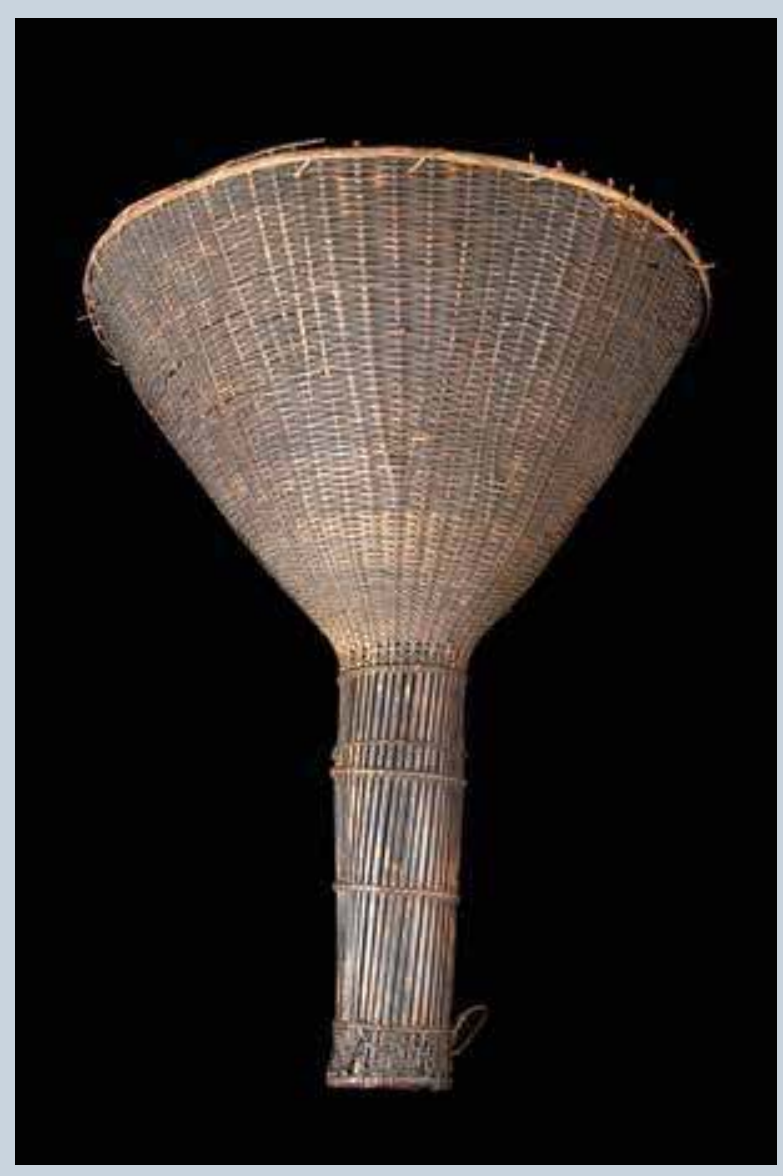

4. Piège à termites, $\mathbf{n}^{\circ}$ inventaire 71.1934.133.77.

Donné par l'Institut Océanographique de Nha Trang (Vietnam), cet objet a intégré les collections du musée de l'Homme en 1934. Selon la fiche descriptive, dont les informations créées lors de l'enregistrement au musée de l'Homme ont été reportées sur la fiche TMS, «la forme de cloche à double fond est surmontée d'un cylindre. Vannerie type tissé toile. Brins minces et serrés, montants un peu plus larges et espacés. Pour le cylindre, vannerie type cordé simple à l'extrémité, les brins prennent les montants deux à deux en dégradé. État moyen. Longueur du cylindre: $31 \mathrm{~cm}$; Diamètre du cylindre: $8 \mathrm{~cm}$ ». 


\section{$\star_{\text {MUSÉE DU QUAI BRANLY - Jacques Chirac }}$}

Notice d'objet

Notice $\mathrm{D}^{\circ} \mathrm{Y}-326579$

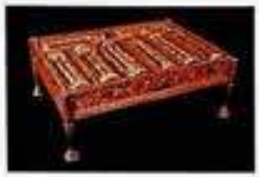

Numbre efimetrain

Units patrimaniale

Appentiation ou tere

Noen veenatulaire

hivteur

Glassification

Datation

Description

Usare

Natiriaux et techingues

Dimsenion:

Teaptoryme:

Ethoratye / 5 t, te

Personinets) et imtaution(s)

Autrefsy numberos:

notes

Culture

Prifiode
$71.1053,15124$

Oolanie

Con in arequets

Porotaryen

\section{thon renseingit.}

Arant 1905

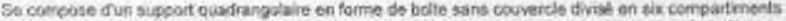

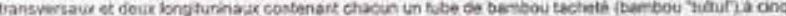

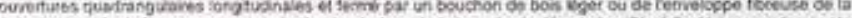

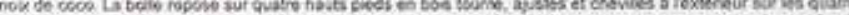

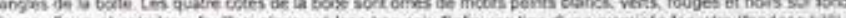

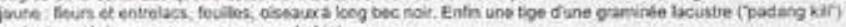

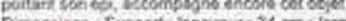

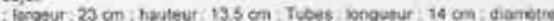

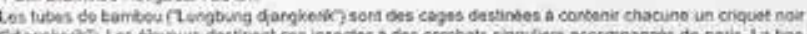

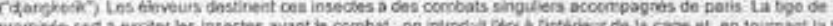

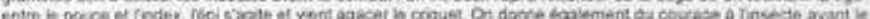

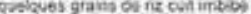
Bon soums es peist berrbou

$13 \times 34 \times 24$ con 537

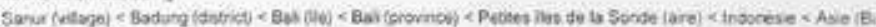
sinen

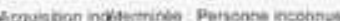

Wovith Leus Berthe

Proctidete cellection Mrste de PHomme iOctanit.

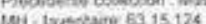


est potentiellement «infesté » par des présences bien réelles qui, pour peu qu'on en contrôle le bruit et l'intensité, ouvrent des possibilités de cohabitation.

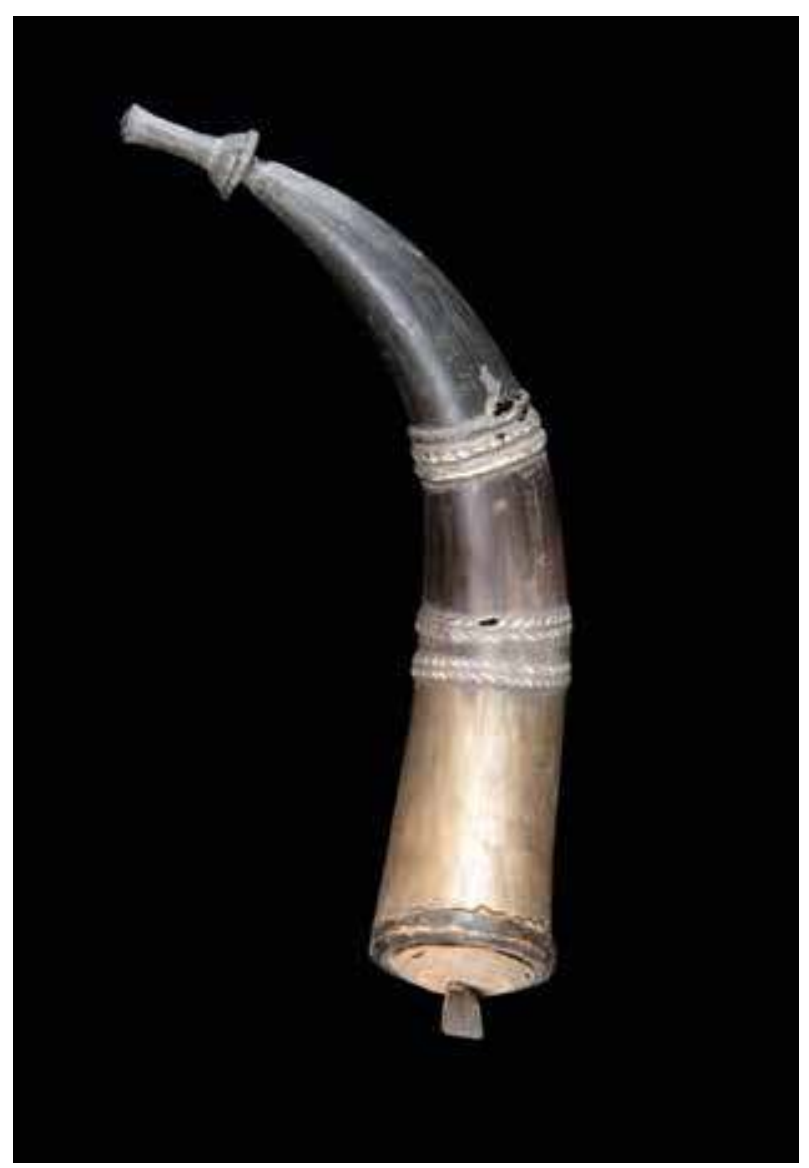

6. Corne à poudre?, $\mathrm{n}^{\circ}$ inventaire 75.15360 .91$.

Objet provenant d'Afrique, , donateur Georges Olivier de Sanderval, précédente collection Musée national des arts d'Afrique et d'Océanie. Sur la fiche TMS objets nous pouvons lire à la rubrique Description: «corne cuir bois, corne ouvragée, couvercle en bois et attache en cuir. Trous d'insectes ».

\section{Poudre, poussière}

Traverser l'espace d'exposition du musée du quai Branly après la lecture de Pour le moins (Dagognet 2009) permet d'expérimenter «la frontière qui sépare le réel de ce qui appartient à la catégorie du "presque rien" ", pour reprendre une interrogation chère au philosophe qui s'était intéressé à l'ontologie a minima, au statut réservé aux "existants impalpables», les pulvérisés qui sont «partout et nulle part» (ibid. : 29).

Dans la pénombre des salles du musée, j'observe des particules saisies par le faisceau de lumière de la lampe frontale qui éclaire une tête de pirogue. Elles virevoltent de tous côtés. L'air ambiant de l'espace d'exposition, même s'il est contrôlé et filtré, présente une pluralité de poussières en suspension qui circulent sans cesse. Parfois, cette volatilité répond à la capacité électrostatique des corpuscules de s'attirer entre eux. Si le taux d'humidité ambiante augmente, certains d'entre eux, par leur capacité plus ou moins hydrophilique, tombent et se posent là où ils atterrissent. Parmi ces particules, certaines sont produites par les humains, épithélium et fibres organiques, d'autres, par les objets. Ces résidus prolifèrent, nuisant à la transparence des vitrines et obligeant à un nettoyage régulier; sédimentant sur ou dans les objets poreux et accélérant leur détérioration.

Mes yeux se posent sur l'étagère illuminée par la lampe béret du chargé de la conservation préventive. Je scrute son geste délicat, le regarde ramasser - ramassage d'un autre ordre - les fragments de matière pulvérisée qui proviennent de l'objet lui-même, ici la tête de pirogue. Ces particules sont ensuite déplacées de l'étagère à l'aide d'un pinceau et conservées dans des sachets en plastique dans une armoire de l'atelier de restauration et de conservation. Elles sont pesées et la variation de masse est contrôlée. Ces fragments pulvérisés deviennent des indicateurs des changements hygrométriques ou 


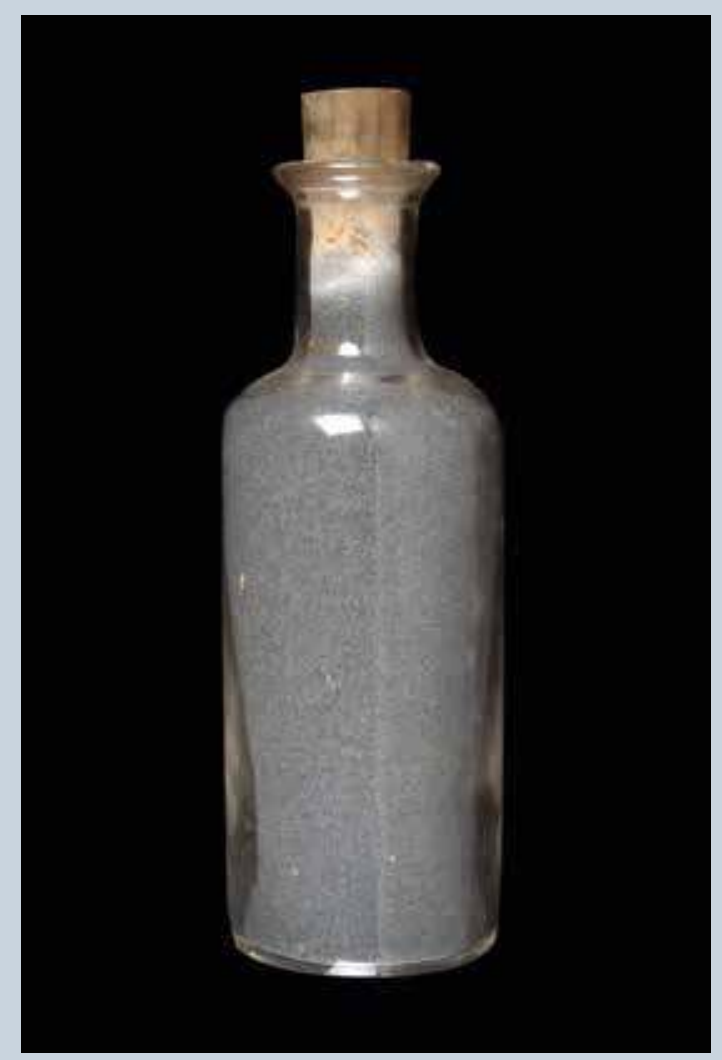

Flacon en verre contenant de la poudre grise.

Il était conservé au musée de l'Homme dans le département d'Asie. Lors de son transfert au musée du quai Branly, aucune trace d'appartenance à une collection n'a été trouvée. Le flacon, le bouchon en liège et la poudre ont été enregistrés au musée par un numéro d'inventaire en $Z$ (Z388065), qui identifie un objet non patrimonial mais qui circule dans la base de données avec les objets enregistrés dans l'inventaire d'État.

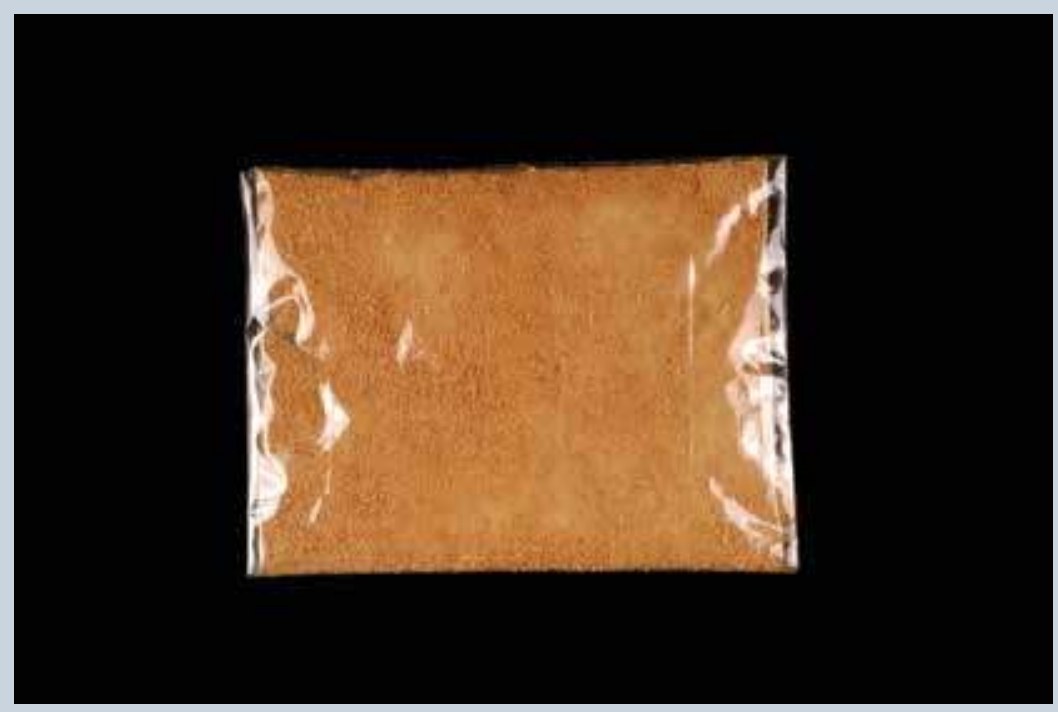

Poudre divinatoire, Ye, poudre de bois conservée dans un sachet, $n^{\circ}$ inventaire 71.1938.17.30.1-2.

Elle a été rapportée par la mission Bernard Maupoil du Bénin, inventoriée au musée de l'Homme en 1938 et transférée au musée du quai Branly. 


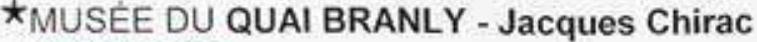 Notice d'objet}

Notice $n^{2}: 209303$

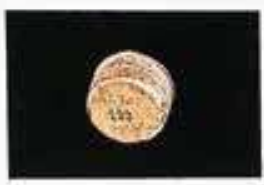

Numére dienventaice

71.1931 .74 .17

Unite patrimoniale

Appolistion ou titre

Nom vernaculs ire

Auteur

Classidication

Dutation

Description

Usage

Motériaux et techniques

Dimensions

Teponyme:

Ethnomme / Style :

Personots et institutionsis

Atrique

Poudre dos

Dond

Inon tenseignd)

avart togt:

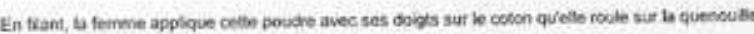

Poussibse ros caiche angiombit avec de leou

$31 \times 3,1 \times 1,8 \mathrm{~cm}, 129$

Seneigat < Atrique occidertaio \& Arique (Aecien cencle de Bake)

Sencopder

Acesusition indiserminte : Persone inconnus

Mission: Mission Dakar Opoul

Piocedente collection : Musbe de Hiomme iadriquei

Autross) numeroits

MH- Inventave: $31.74,17$ ?

Notes

Sentgat

Cunture

Periods 
de suspicion d'infestation. Cette poussière de bois constitue ainsi un échantillon de résidus de l'objet exposé en relation à son environnement. Observer ce déplacement des fragments pulvérisés, nous permet de ramener la tête de pirogue à «la vie animée», pour reprendre une expression d'Ingold ([2013] 2017).

Cet échantillon recueilli sur le plateau d'exposition côtoie, dans les réserves, tout un tas d'autres prélevés par les ethnologues sur leur terrain respectif: ensemble de matières conçues comme représentatives, racontant à leur manière quelque chose des populations de provenance. Parmi ces échantillons, les poudres sont nombreuses: poudres d'os, d'arachides grillées, de graines diverses, de piments, de charbon, de cendres, de bois, d'écorce, de salpêtre, de soufre, de terre, d'or, de calcite, de pierre... Toutes, qu'elles soient minérales, végétales ou animales, sont broyées, pilées, composites, formant des tas de couleur brune, ocre, rouge, blanche, rose, grise. Elles existent en tas, car le seul fragment disparaît à l'œil humain et ne permet aucune saisie de quelconque activité humaine ou non humaine.

Ces fragments pulvérisés sont utilisés pour la divination, pour des rites funéraires, pour des fêtes ou comme porte-bonheur. Ils participent à la constitution du réel observé et permettent d'établir une communication ou un relais entre des ordres de grandeur autrement incommensurables - les humains et les dieux par exemple. Ce pouvoir leur est conféré par leur caractère perméable et non structuré (Holbraad 2007). D’autres poudres aident à guérir ou à prévenir les maladies; d'autres encore servent à filer, à préparer des aliments, à épiler, à colorer des visages, des tissus, à dégraisser la peau ou la poterie. Elles agissent parce qu'elles sont poreuses, palpables, absorbantes et frottantes.

Concassées, les substances gagnent en pouvoir d'accrochage ou de mélange et de transformation. Dans le même temps cependant, elles gagnent en pouvoir de dispersion - d'où la nécessité d'un récipient pour contenir l'état corpusculaire obtenu par pulvérisation. À une pluralité de poudres répond aussi une pluralité de contenants, éprouvettes du monde des sciences naturelles, réalisés par les utilisateurs de ces poudres ou toute sorte de boîte à disposition par le collecteur sur le terrain.

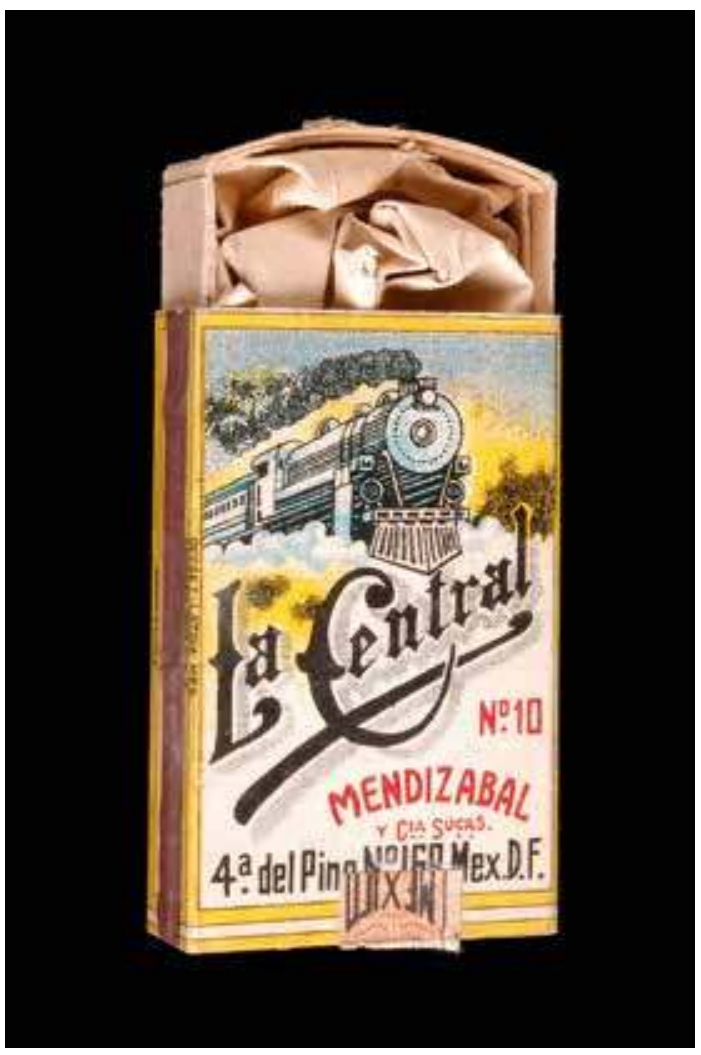

10. Échantillon de dégraissant, $n^{\circ}$ inventaire 71.1937.24.67, rapporté lors d'une mission à San Pedro de las Anonas (Mexique) par Guy Stresser Péan.

II s'agit de la poudre de la pierre «chay» (calcite) utilisée pour dégraisser la poterie.

L'hétérogénéité de ces poudres et poussières, qu'elles soient échantillons d'altérité (et collection d'ethnographie) ou de relation à l'environnement de conservation, contribue à tisser un musée qui, vu d'en bas, ne cesse de se transformer. 


\section{Fibre}

Je découvre la fibre par le détour du papier, ou plus exactement de celui du colmatage des minuscules trous laissés par des larves d'insectes xylophages dans un manuscrit ancien. La restauration manuelle des ouvrages s'effectue à l'aide de papier japon, un papier imitant le papier washi, produit au Japon à partir de la fibre d'écorce, le plus communément de mûrier. Racha m’a montré comment émietter de petits morceaux correspondant à la taille des trous à combler, puis à adjoindre progressivement le papier de restauration au papier du manuscrit, au moyen d'un scalpel avec lequel sont grattés doucement les bords. Le papier japon est le papier privilégié par les restaurateurs en raison de ses propriétés à la fois de souplesse et de résistance, qui rendent sa longévité exceptionnelle. C'est un papier très fibreux, dont j'apprivoise peu à peu la texture particulière: les filaments qui émergent progressivement sous les raclements légers de la lame du scalpel, et qu'il faut marier graduellement à ceux, presque invisibles, du feuillet à restaurer. Mes propres gestes, aussi méticuleux que maladroits, contrastent avec ceux, rapides et automatiques, de Racha et des autres restauratrices qui m'entourent: rompues au maniement délicat du scalpel, elles dégagent sans presque y prêter attention les fins filaments des bords filandreux du papier, habituées qu'elles sont à percevoir, sous le grattage de la lame, la résistance des brins qu'il faut libérer sans les rompre.

Entrée «fibre» donc, un rappel de ma découverte tactile des propriétés de la fibre, avec ses deux caractéristiques majeures: celle en premier lieu de se plier, en raison de sa structure physique, de manière unique au travail humain: les fibres peuvent être nouées, tissées, tressées, broyées, écrasées, nattées. François Dagognet nous le rappelle à propos du textile, la fibre est un moteur essentiel de l'innovation humaine, par ses qualités uniques de souplesse et de résistance, de légèreté et de solidité, qualités qu'on n’aura cessé de travailler, améliorer, imiter, synthétiser; elle est un des lieux où s'est jouée une bataille «technologique et philosophique » pour vaincre la nature (Dagognet 1989).

La fibre a, seconde caractéristique, la capacité de littéralement se fondre dans d'autres matières: le papier, assemblage de fibres entremêlées et de colle, en est l'exemple le plus évident. Un autre, qui resta longtemps énigmatique, est celui du fil de soie: dans son histoire de l'industrie textile et son effort de fabriquer autrement les propriétés de la fibre, Dagognet raconte que Réaumur s'évertue à essayer de comprendre comment, à travers la médiation du ver, la fibre végétale est transformée en fibre animale, la feuille de mûrier en fil de soie. Dans ses observations du ver à soie et ses tentatives de décomposer les étapes de cette métamorphose, Réaumur «ouvre un domaine assez neuf, l'ensemble de la mésomorphie, celui de tous les produits à la fois solides et liquides - la colle, les résines, les laques, les mucilages, les gommes. Nous avons été trop influencés soit par les solides eux-mêmes, soit par les fluides qu'on dispose d'ailleurs dans des récipients étanches, soit encore par les grains et les poussières. Enfin, par Réaumur, nous sommes mis en présence de ces corps intermédiaires, plus tard, la nébuleuse s'élargira avec les gels, les adhésifs et les cristaux solides. (Ibid. : 112-113).

L'entrée «fibre» fait cheminer dans un assemblage composite, entre différentes alternatives d'utilisation et de travail et transformation - papier, cordelette, tissu, natte, tresse, vannerie... 


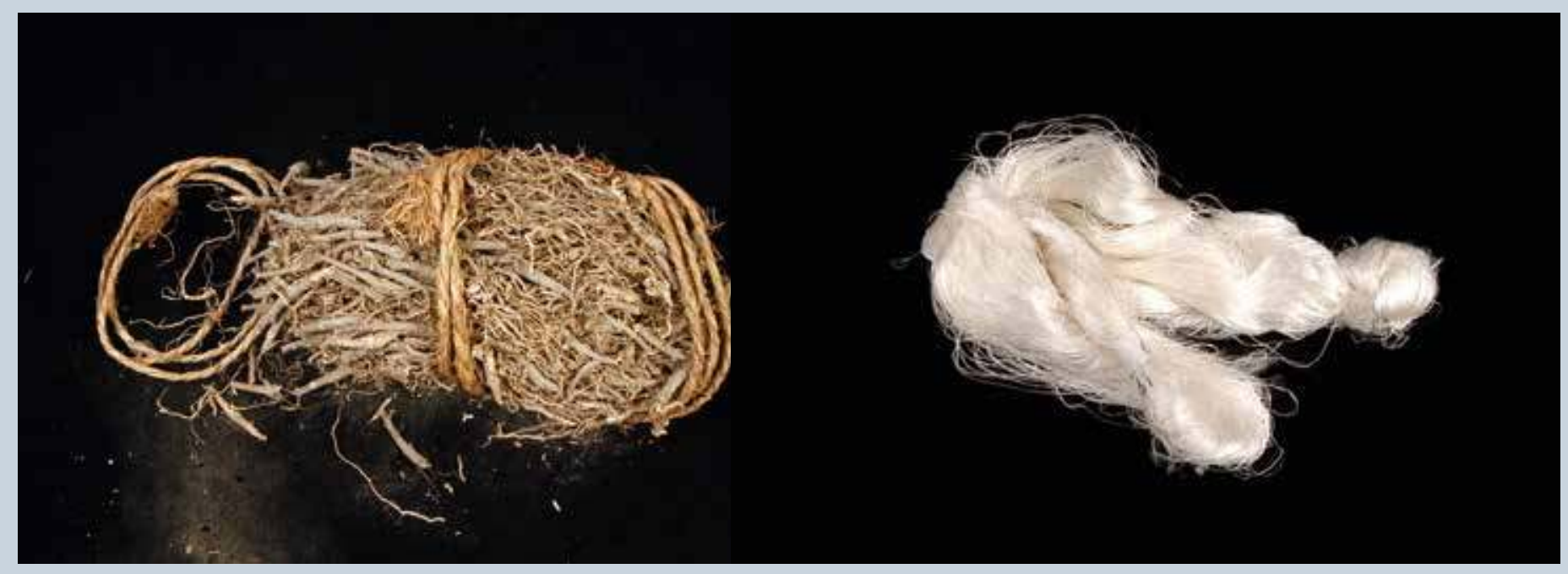

11. Matériaux: fibre végétale, $\mathrm{n}^{\circ}$ inventaire 71.1947.51.390.

«Matière brute (branches) pour la fabrication du papier. Traces d'infestation » précise la notice à propos de cette «matière à papier » rapportée par André Leroi-Gourhan de son séjour japonais, sans doute des racines de tororo aoi dont l'excrétion est utilisée pour lier la pulpe de papier obtenue à partir des fibres d'écorce d'arbre.

\section{Matériaux: fibre animale, $n^{\circ}$ inventaire 71.1969.19.10.}

Écheveau de soie, Classification: Objet. La fiche donne son origine géographique l'île de Sulawesi en Indonésie, l'auteur de la mission (Christian Pelras). Les champs Description et Usage sont vides.

\section{Exemple de notice d'objet. Amulette}

Numbro dinventaire

\section{Unite parrinenside}

Appettation ou titro

Nors vernaculate

Auteur

Ciassification:

Datation

Description

Usage:

Matoriaux et techniques

Dimeersions

Toperyme :

Eihnonyme istyle

Personne(s) st institution(s)

Autre(s) mumbrots)

Notes

Culture

periode

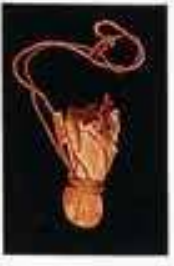

7193372734

Amernies

Amibese

Textio ou vitemion

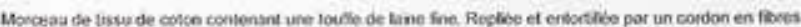
veghoses lengueut icns

Coton tisos, taine, tore vidgetale thossece.

$8 \times 5 \times 2,5 \mathrm{~cm}, 8 \mathrm{~g}$

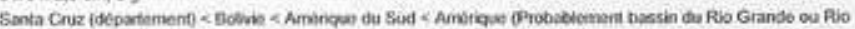

Guspay

Acquistion ind bensinte : Personne inconnos

Mosion: Alted Mitran

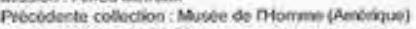

19H- hwelaire 3372.734

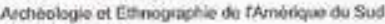

non renseligeto

nos renseignte. 


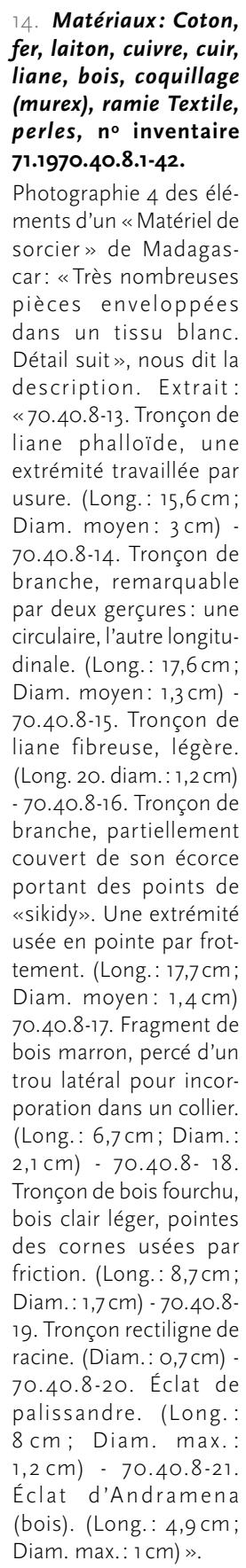

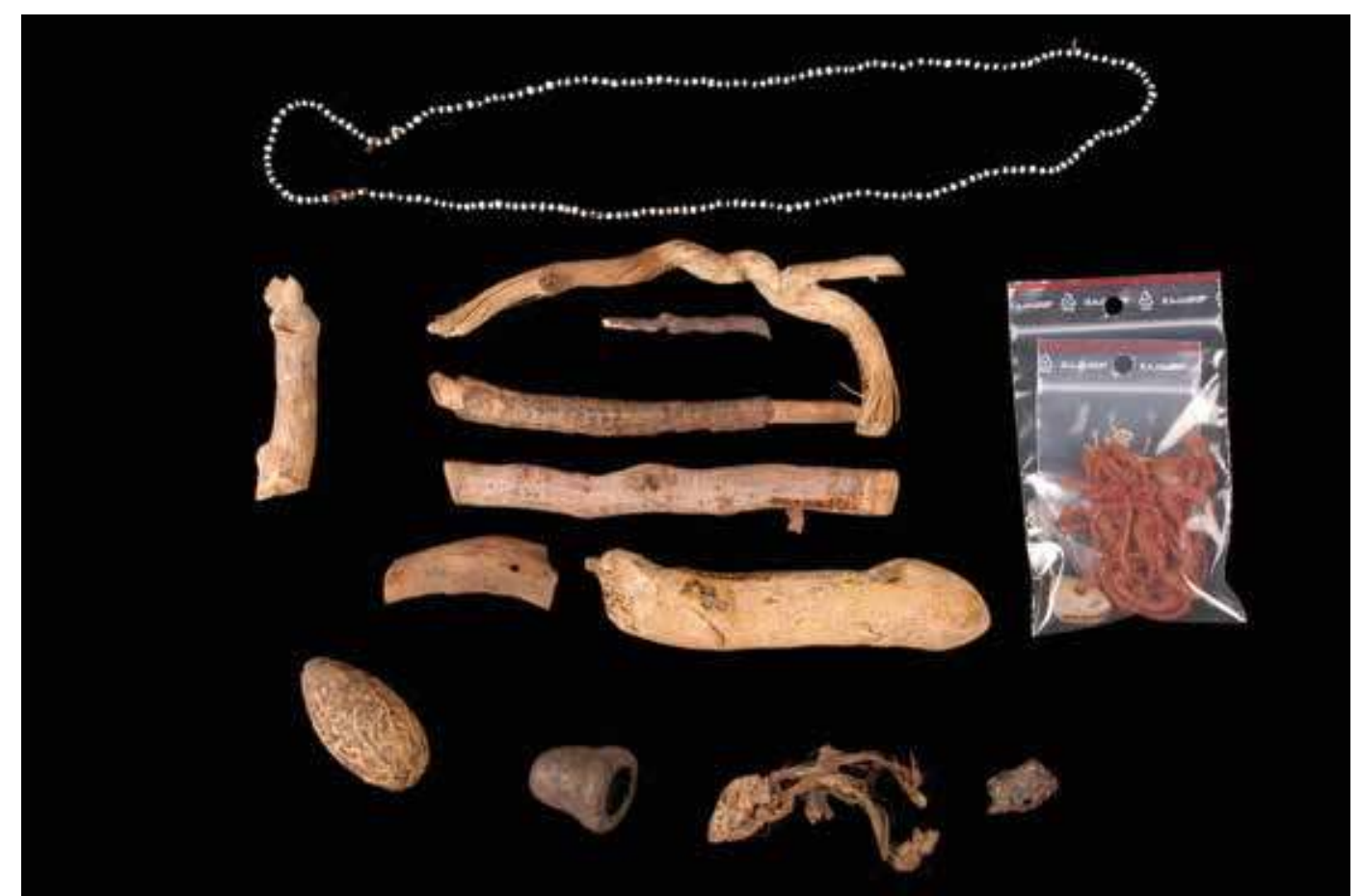

soit l'étendue des productions techniques que la fibre, simultanément «bonne à manier » et «bonne à penser», a permis de déployer. Elle rend compte alors, très pratiquement, de la difficulté rencontrée par ceux qui ont rempli les fiches des objets des collections dans leur effort de décrire correctement les objets collectés: le «matériau» est-il de la soie (ou de la laine) ou de la fibre animale? Dans quel degré de précision de la composition matérielle faut-il entrer? Jusqu'à quelle échelle descendre? «Cordelette» dira l'un, « fibre végétale » mentionnera un deuxième, «cordelette fibreuse» tranche un troisième quand il faut nommer cordes ou ficelles. L'entrée « fibre » nous emmène enfin, par le biais d'un hermétique «tronçon de liane fibreuse », dans un objet à description impossible: un " "matériel" pour fabriquer des ody ou mohara (amulettes)», comme le note la notice à propos d'un agglomérat de petites pièces issu de la collection Madame William Lods, recueillie lors d'une mission protestante à Madagascar vers les années 1950. Sont mêlés ici boutons, bouchons et lianes, perles, écorces, débris et tronçons de fil de fer. Toutes les pièces sont méticuleusement numérotées, mesurées, décrites les unes après les autres. La description laisse poindre l'impératif de la connaissance la plus exacte que vient troubler la perplexité: «magma solide, composition encore inconnue» est indiqué, avec optimisme et hésitation, à propos d'un petit truc qui se niche dans un bouchon en bois - comme si la bataille «philosophico-technique» de la maîtrise du monde se jouait, aussi, ici. 


\section{Concrétion}

Soit, pour finir, un caillou. Ramassé sur une plage d'un endroit qui vous est cher, peut-être, vous l'avez d'abord glissé dans votre poche et caressé sans y penser - quoique son toucher vous extirpe toujours un peu du flot d'actions et de sensations dans lequel vous êtes pris et vous amène vaguement ailleurs. Vous l'aurez oublié peut-être, puis retrouvé un jour au moment de remettre la veste l'hiver suivant. Il rejoindra alors peut-être le récipient dans lequel vous avez déposé au fil des années, faute de pouvoir jamais les jeter, d'autres cailloux du même genre acquis dans des circonstances analogues, ou bien gagnera le privilège de trôner un temps sur votre bureau ou votre bibliothèque. Vous le regarderez de temps à autre, y verrez chaque fois de nouveaux détails, de nouvelles couleurs, de nouveaux reliefs. Vous pourriez vous y perdre: d'imaginer le temps nécessaire à sa formation, incommensurable avec le vôtre; d'entrevoir par son entremise les dynamiques écologiques massives en même temps que dérisoires dont il se fait le témoin. Parfois, vous ressentez une sorte de vertige (Morton 2013).

Les archéologues le savent bien - les spécialistes de la «microchronologie » plus encore: les concrétions et sédiments constituent des agrégats compliqués qui requièrent beaucoup d'interprétations (Petit et al. à paraître). Qui, autrement dit, mettent au travail ceux qui les regardent. Il faut défaire, ne serait-ce que par la pensée, l'imbroglio d'agents naturels qui les constituent, auquel sont venus s'ajouter même parfois des agents non naturels - une présence humaine, pourquoi pas, qui aurait fait quelque chose dont l'image ou le sens nous seraient à présent devenus opaques. Les concrétions, les encroûtements, les cristallisations, sont utilisés par les archéologues comme des «enregistreurs continus du temps» : ils leur permettent de définir « une très haute résolution temporelle», dans laquelle se lisent des séries de micro-événements qui se suivent -au point de sédimenter. Pour peu qu'on daigne y prêter attention, ils interrogent: que s'est-il passé?

La plus petite de ces pierres ramassées sur la plage ou en forêt, dans son impureté même, raconte donc potentiellement des tas d'histoires et recèle d'innombrables potentialités. C'est ce qui intéressait les maniéristes du $\mathrm{XvI}^{\mathrm{e}}$ siècle, qui s'extasiaient des potentialités de la «matérialité inerte»: dans les «collisions» innombrables qu'elle rendait manifestes, elle leur permettait de «scruter les effets d'une genèse ou d'un dépérissement» (Falguières 2004: 55). Comme si les maniéristes prenaient acte, au sens fort du terme, des propositions édictées dans le traité de la Génération et de la corruption, constituant le livre IV des Météorologiques d'Aristote, qui, suivant les termes de Patricia Falguières, «se déploie en une vaste et minutieuse enquête sur les qualités des corps, selon les différents degrés d'agrégation de la matière. [...] À l'extraordinaire collection de matériaux présentée par les Météorologiques - huile, pierre ponce, pyrimaque, chaux, boue, argile, nitre et cristaux de sel, vin, urine, vinaigre, miel et cendre, petit-lait et j'en passe - répond un arsenal d'opérations -coction, maturation, ébullition, rôtissage, échaudage, dessiccation, fusion et liquéfaction, etc. - introduites ex abrupto dans le discours philosophique.» (p. 61).

Remarquables par leurs formes (irrégulières voire tourmentées, allongées et étranglées, branchues, mamelonnées...) ou bien par leurs effets (fixer, guérir, repousser, protéger...), les 
Matériaux: Pierre, $\mathrm{n}^{\circ}$ inventaire 71.1965.102.82.

La description et l'usage donnent: «Concrétion minérale blanchâtre servant de fixateur pour teinture ». Mission: Corneille Jest. Ces « [cinq] fragments de concrétion» tibétains ont été enregistrés au musée de l'Homme (département Asie) en 1965.

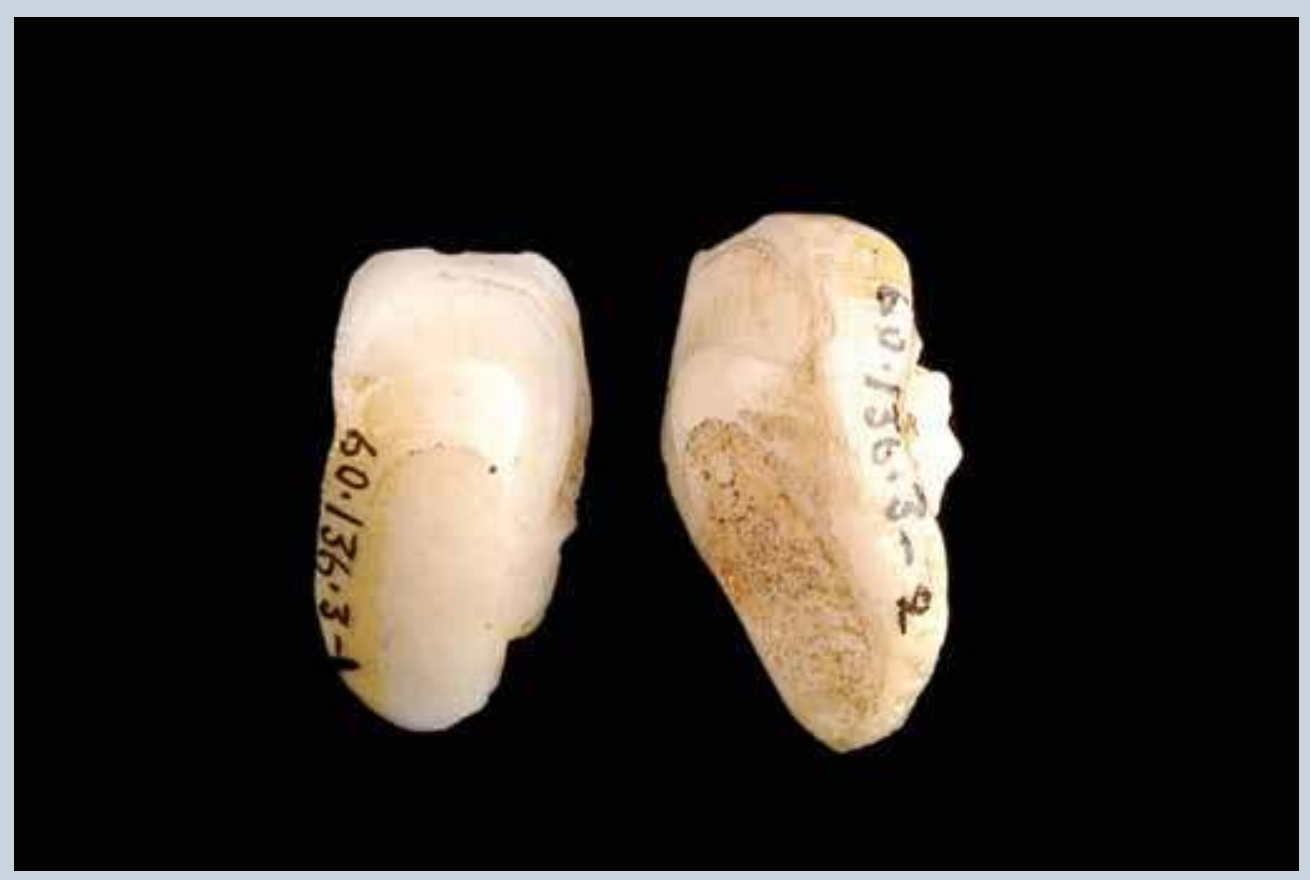

\section{$\star_{\text {MUSÉE DU QUAI BRANLY - Jacques Chirac }}$}

Notice d'objet

\section{Notice $n^{\prime \prime}$ : 8428}

16. Concrétion «très tourmentée» de magnésie, dit la fiche.

C'est le tourment de la magnésie, la forme ramassée sur elle-même, les ciselures chaotiques, qui rappellent aux Kanaks l'aspect des nuages et, «de ce fait», précise la fiche, «est employée comme pierre à pluie dans la culture humide».

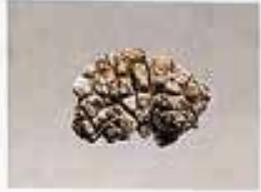

Numero dinventaire

Unite patrimeniase

Aspellation ou titre

Nom vernaculaire

Auteur

Classification

Datation

Description

Usage

Nateriaux en techniques

Dimensicens

Toporyene :

Ethnomme/stipte

Personne(s) et iestituation(s)

Autre(s) numiro(s)

Notes

Culture

Periode
7). 182030.23

Oseanie

Phere a mage

inson reviseignel

miliev tee debut ade rectiv

Cenoweion trits beumemise de manesie.

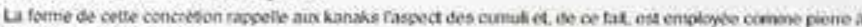
plute dans le cille himide

Lathique

$15 \times 10.5 \times 5 \mathrm{~cm} \cdot 841 \mathrm{~g}$

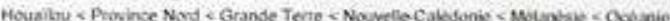

Kanges

Conmeur: Mounce Leenthard

Precodents solection: Mubie de nionime (Dotanisy

MET- Monbine etig

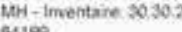




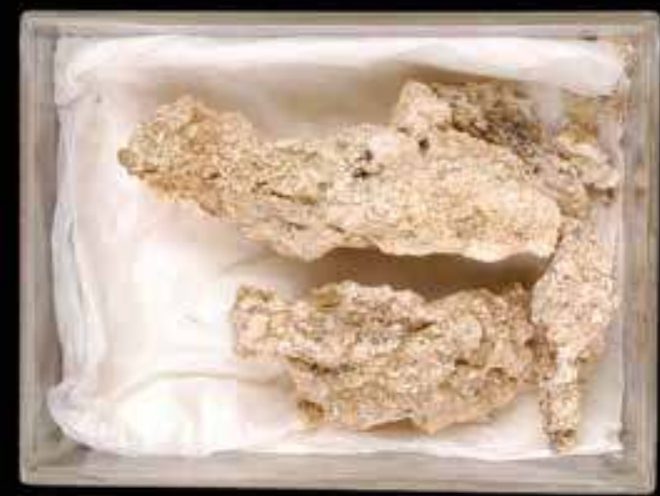

Matériaux: otolithe,

$\mathrm{n}^{\circ}$ inventaire 71.1960.136.3.1-2.

Concrétion naturelle de poisson (pierre d'oreille) d'un blanc laiteux et de forme irrégulière. 1) longueur: $2,8 \mathrm{~cm} ; 2$ ) longueur: $3 \mathrm{~cm}$. Donateur au musée de l'Homme (Afrique du Nord et ProcheOrient) : Ernest Gustave Gobert.

«Élément prophylactique. L'origine marine des otolithes et leur forme qui se prête à des symbolismes divers en font des phylactères particulièrement estimés des Tunisiens depuis l'Antiquité. On les trouve dans les portefeuilles ou les porte-monnaie, tels quels; pilés et mêlés à du miel, ils sont employés pour combattre les affections urinaires et la coqueluche. »

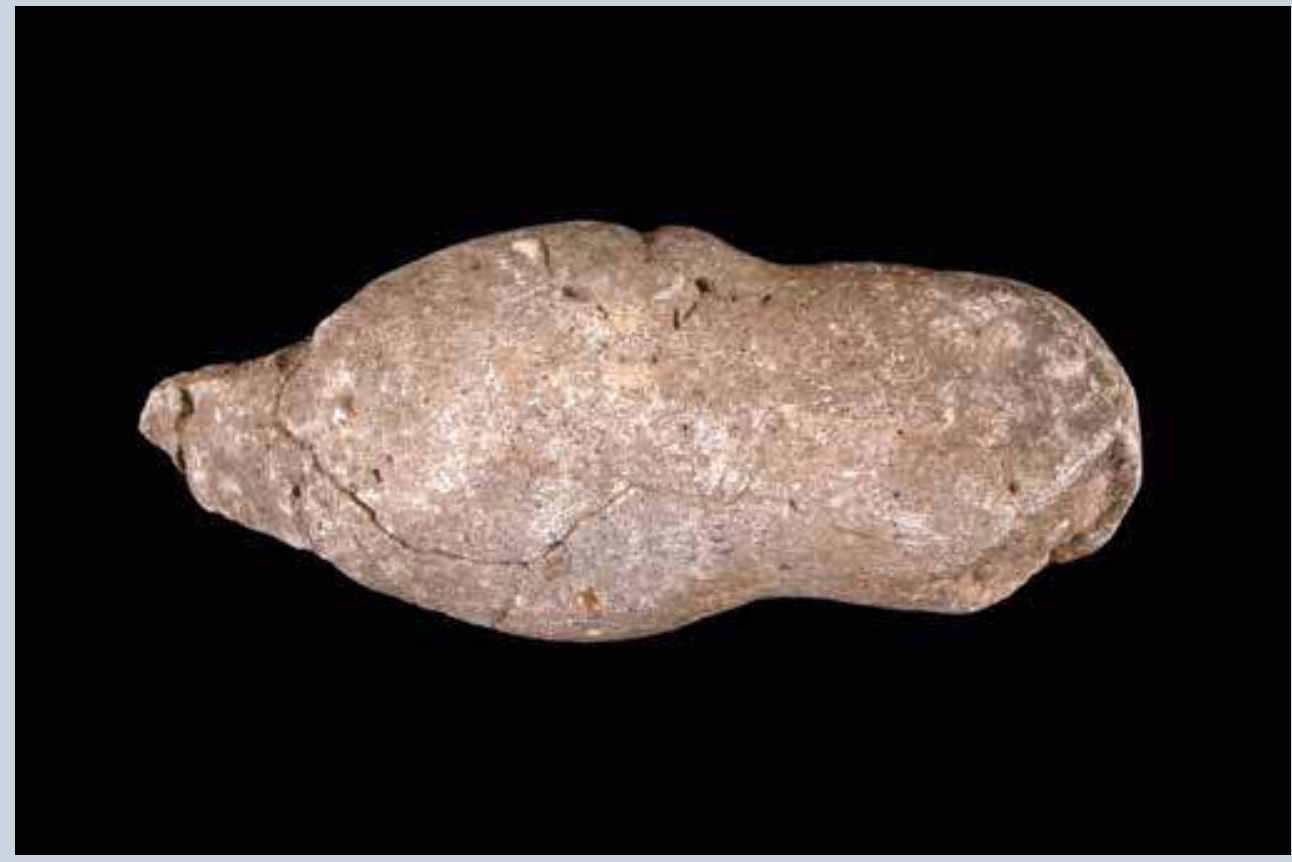

\section{Matériaux: pierre argile de Koumac, $\mathrm{n}^{\circ}$ inventaire 71.1931.50.16.}

La description donne: «Concrétion calcaire allongée, étranglée au $1 / 3$ et effilée à une extrémité. Cupule d'éclatement à l'autre extrémité qui est arrondie. L. =14,2; Larg. max. =5,7》. Quant à l'usage, la fiche stipule: « Cette pierre est envoyée par le donateur comme "sorte" d'argile mangée par les géophages ». « ll y a certainement erreur», poursuit diplomatiquement le rédacteur de la fiche, «la roche étant un calcaire fort dur dans lequel se voient des débris de coquilles. Il y a tout lieu de la considérer comme pierre magique, la forme, bien que peu caractéristique, la rapproche des pierres pour l'igname». Donateur au musée de l'Homme (Océanie): Philippe Rey-Lescure. 
concrétions ont une consistance singulière qui les apprête à des usages extrêmement divers et souvent équivoques - c'est tout leur intérêt. Elles sont le support d'analogies efficaces (telle concrétion rappelant aux Kanaks un cumulus) ou bien de pratiques prophylactiques mettant à profit des connaissances souvent complexes des effets plus ou moins subtils qu'une matière peut produire sur un corps, une partie de corps, un organe. Mais, comme pour les poudres, comme pour les fibres, un trouble souvent persiste sur la place qu'occupaient ces cristallisations ou conglomérats, et la sorte de contrat qui liait chacun à une personne ou à une communauté de personnes. Des histoires se racontent, s'éprouvent aussi par les ethnographes, les collectionneurs ou les conservateurs, pour mettre des mots et stabiliser, ne serait-ce que provisoirement, ce qui distingue celle-là ou celui-ci de n'importe lequel des cailloux ramassés sur la plage.

\section{Les auteurs}

Tiziana N. Beltrame, chercheure postdoctorante en anthropologie; ses centres d'intérêt et sa recherche portent sur les pratiques de conservation des objets muséaux, numériques et physiques. Elle s'interroge notamment sur la création des temporalités des œuvres et sur l'instabilité des matériaux du patrimoine culturel et scientifique. Elle est actuellement postdoctorante IFRIS au Centre Koyré à Paris (2017-2019).

Sophie Houdart est anthropologue, directrice de recherche au CNRS, Laboratoire d'ethnologie et de sociologie comparative (LESC). Spécialisée sur le Japon, elle a réalisé plusieurs enquêtes sur le thème de la création et de l'innovation, dans les champs des sciences, de l'art ou de l'architecture. Elle travaille aujourd'hui sur la vie après la catastrophe de Fukushima.

Christine Jungen est anthropologue, chargée de recherche au CNRS, Laboratoire d'ethnologie et de sociologie comparative (LESC). Elle s'intéresse, à partir du Moyen-Orient, au goût pour les vieux papiers, aux environnements matériels de l'histoire, à l'érudition et ses savoir- faire ainsi qu'aux mises au format du passé.

Après une thèse sur l'histoire de l'anthropologie dans ses rapports avec la philosophie, Frédéric Keck a mené des recherches sur la grippe aviaire dans le cadre du Laboratoire d'anthropologie sociale au CNRS, et dirige actuellement le département de la recherche et de l'enseignement du musée du quai Branly.

\section{Iconographie}

Image d'ouverture. (C) musée du quai Branly - Jacques Chirac / photo: Thierry Ollivier, Michel Urtad.

1. et suivantes. (C) musée du quai Branly - Jacques Chirac.

\section{Bibliographie}

Bondaz, J. 2013 «L'ethnographie parasitée? Anthropologie et entomologie en Afrique de l'Ouest (1928-1960)», L'Homme 206(2): 121-150.

Dagognet, F. 1989 Rematérialiser. Paris: Vrin.

- 2009 Pour le moins. Paris, Vrin.

16. (C) musée du quai Branly - Jacques Chirac / photo: Thierry Ollivier, Michel Urtad.

Falguières, P. 2004 «Extase de la matière. Notes sur la physique des maniéristes», in $\mathrm{H}$. Brunon, M. Mosser \& D. Rabneau (dir.), Les éléments et les métamorphoses de la nature. Imaginaire et symbolique des arts dans la 
culture européenne du XVI $I^{e}$ au XVIII siècle. Bordeaux, Paris: William Blake \& Co/Art \& Arts.

Haraway, D. 2016 Staying With the Trouble: Making Kin in the Chthulucene. Durham: Duke University Press.

Holbraad, M. 2007 «The Power of powder: Multiplicity and notion in the divinatory cosmology of Cuban Ifá » in A. Henare, M. Holbraad \& S. Wastell (dir.), Thinking Through Things: Theorising artefacts ethnographically. New York: Routledge.

Ingold, T. 2017 [2013] Faire Anthropologie, archéologie, art et architecture. Bellevaux: Éditions Dehors.
Morton, T. 2013 Hyperobjects: Philosophy and Ecology after the End of the World. Minneapolis, Londres: University of Minnesota Press.

Petit, C., Brochier, J.-E., Curie, J., Desachy, B. et al. 2018 (à paraître) «À la recherche du temps court : l'approche microchronologique en archéologie. Étude des encroûtements carbonatés en contexte paléolithique et romain» in C. Andrieu \& S. Houdart (dir.), La composition du temps, Actes du 14e colloque annuel de la MAE.

\section{Pour citer l'article}

Beltrame, T. N., Houdart, S., Jungen, C. \& F. Keck 2017 «Sonder une collection», Techniques\&Culture 68 « Mondes infimes», p. 178-195. 